\title{
On the Measurement of the Stability Derivatives by means of Forced Yawing Technique
}

\author{
By Seizo Motora, Member* \\ Masataka Fujino, Member
}

\begin{abstract}
Summary
In this paper, the authors describe the forced yawing technique, especially the mechanism of the yawing oscillator and measuring system, by which the stability derivatives of a ship model are measured.

In order to compare the derivatives obtained by this method with the ones obtained by the rotating arm technique, the authors carried out the experiments in low frequency range. As a result of these tests, a fairly well agreement between both data was recognized.

In addition, the authors discuss that what kinds of motions are to be given to a ship model in order to get data more accurately and easily.
\end{abstract}

\section{Introduction}

In dealing with the response of ships to periodical rudder movement or disturbance caused by waves, it becomes necessary to evaluate the stability derivatives as frequency dependents. For this purpose, a forced yawing technique is thought most suitable. This technique also has a merit that it is applicable in regular towing tanks. The forced yawing technique has been used at DTMB ${ }^{1}$ for the problems of depth control of submerged bodies. This technique was applied to measure the stability derivatives of a surface ship in restricted waters at the University of Michigan ${ }^{2}$. In this case, only a combined motion which will be stated later was used.

The same technique was tested by Paulling ${ }^{3)}$ and Van Leeuwen ${ }^{4)}$ in more refined ways showing that

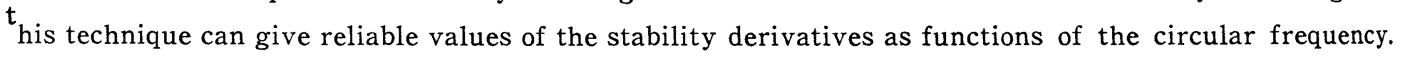
A refined type of oscillator ${ }^{5}$ ) based on the one which was used by Motora and Couch at the University of Michigan has been constructed at the University of Tokyo and has been working since the middle of 1964. A ship model of the series $60, \mathrm{Cb}=0.60$ of which the stability derivatives were obtained by the rotating arm technique at Stevens Institute of Technology was tested by this oscillator. In this paper, the authors intend to show the correlation between the stability derivatives obtained by the forced yawing technique and the ones obtained by the rotating arm technique.

\section{Principle of the forced yawing technique}

Using a co-ordinate fixed at the C.G. of a ship, as shown in Fig.1, a linearized equation of motion is expressed as follows :

$$
\left.\begin{array}{l}
-\left(m+m_{y}\right) U \dot{\beta}+m_{y} \alpha \dot{r}=Y_{\beta} \cdot \beta+\left\{-\left(m+m_{x}\right) U+Y_{r}\right\} r+Y_{0} \\
\left(I_{z}+J_{z}\right) \dot{r}-m_{y} \alpha U \dot{\beta}=N_{\beta} \cdot \beta+N_{r} \cdot r+N_{0}
\end{array}\right\}
$$

where

$U$ : speed of advance

$\beta$ : drift angle

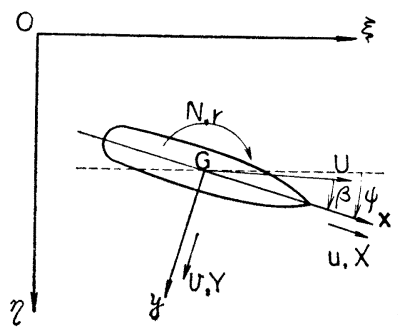

Fig. 1 Co-ordinates System 
$r$ : yaw angular velocity

$m+m_{y}:$ virtual mass to the $y$-axis direction

$I_{z}+J_{z}$ : virtual mass moment of inertia about $z$-axis

$Y:$ hydrodynamic lateral force on the ship, $Y_{\beta}=\frac{\partial Y}{\partial \beta}, Y_{r}=\frac{\partial Y}{\partial r}$

$N$ : hydrodynamic moment on the ship about $z$-axis, $N_{\beta}=\frac{\partial N}{\partial \beta}, N_{r}=\frac{\partial N}{\partial r}$

$Y_{0}, N_{0}$; external force and moment delivered to the ship by the oscillator

$\alpha$ : longitudinal distance between the centre of added mass, $m_{y}$, and C. G. of the ship

(i) Pure swaying motion

In this motion a ship model is forced to sway without yawing motion. Therefore $\beta$ only changes periodically. Denoting the force and moment which are necessary to confine the ship on this motion as $Y_{0}$ and $N_{0}$, and the phase shift of $Y_{0}$ and $N_{0}$ to the motion as $\varepsilon_{1}$ and $\varepsilon_{2}$ respectively, we get:

$$
\left.\begin{array}{l}
\beta=\bar{\beta} \cos \omega t \\
r=0 \\
Y_{0}=\bar{Y}_{0} \cos \left(\omega t-\varepsilon_{1}\right) \\
N_{0}=\bar{N}_{0} \cos \left(\omega t-\varepsilon_{2}\right)
\end{array}\right\}
$$

Substituting (2) into (1), we get :

$$
\left.\begin{array}{l}
Y_{0}=\left(m+m_{y}\right) U \bar{\beta} \omega \sin \omega t-Y_{\beta} \bar{\beta} \cos \omega t \\
N_{0}=m_{y} \alpha U \bar{\beta} \sin \omega t-N_{\beta} \bar{\beta} \cos \omega t
\end{array}\right\}
$$

Equating (2) and (3), we can get the in-phase and out-phase components of $Y_{0}$ and $N_{0}$.

$$
\left.\begin{array}{ll}
Y_{\beta}=-\frac{\bar{Y}_{0} \cos \varepsilon_{1}}{\bar{\beta}}, & m+m_{y}=\frac{\bar{Y}_{0} \sin \varepsilon_{1}}{\bar{\beta} \omega U} \\
N_{\beta}=-\frac{\bar{N}_{0} \cos \varepsilon_{2}}{\bar{\beta}}, & m_{y} \alpha=\frac{\bar{N}_{0} \sin \varepsilon_{2}}{\bar{\beta} \omega U}
\end{array}\right\}
$$

From (4), we can get $m+m_{y}, Y_{\beta}, N_{\beta}, m_{y} \alpha$ for the specified frequency at which the model was forced to sway.

(ii) Pure yawing motion

In this motion the model is forced to yaw and sway so that the drift angle $\beta$ is always kept to be zero.

Therefore :

$$
r=\bar{r} \cos \omega t, \quad \beta=0
$$

Substituting (5) into (1) we get :

$$
\left.\begin{array}{l}
Y_{0}=-m_{y} \alpha \bar{r} \omega \sin \omega t-\left\{-\left(m+m_{x}\right) U+Y_{r}\right\} \bar{r} \cos \omega t=\bar{Y}_{0} \cos \left(\omega t-\varepsilon_{1}\right) \\
N_{0}=-\left(I_{z}+J_{z}\right) \bar{r} \omega \sin \omega t-N_{r} \bar{r} \cos \omega t=\bar{N}_{0} \cos \left(\omega t-\varepsilon_{2}\right)
\end{array}\right\}
$$

Similar to the previous case, we get:

$$
\left.\begin{array}{l}
-\left(m+m_{x}\right) U+Y_{r}=-\frac{\bar{Y}_{0} \cos \varepsilon_{1}}{\bar{r}}, \quad m_{y} \alpha=-\frac{\bar{Y}_{0} \sin \varepsilon_{1}}{\bar{r} \omega} \\
N_{r}=-\frac{\bar{N}_{0} \cos \varepsilon_{2}}{\bar{r}}, \quad I_{z}+J_{z}=-\frac{\bar{N}_{0} \sin \varepsilon_{2}}{\bar{r} \omega}
\end{array}\right\}
$$

(iii) Combined motion

All the unknown terms in the equation (1) can be obtained by (i) and (ii). However the latter is found to be quite delicate and contain many causes of scatter of data.

Therefore a combined motion is tried to use in place of the pure yawing motion. In the combined motion used in this case, a model is forced to yaw around its center of gravity which runs on a straight course. i. e. :

$$
r=\bar{r} \cos \omega t, \quad \beta=\bar{\beta} \cos (\omega t-\pi / 2), \quad \bar{r}=\bar{\beta} \omega
$$

Substituting (8) into (1) we get 


$$
\begin{aligned}
& \bar{Y}_{0} \cos \varepsilon_{1}=-\left(m+m_{y}\right) U \bar{\beta} \omega-\left\{-\left(m+m_{x}\right) U+Y_{r}\right\} \bar{r} \\
& \bar{Y}_{0} \sin \varepsilon_{1}=-m_{y} \alpha \bar{r} \omega-Y_{\beta} \bar{\beta} \\
& \bar{N}_{0} \cos \varepsilon_{2}=-m_{y} \alpha U \bar{\beta} \omega-N_{r} \bar{r} \\
& \bar{N}_{0} \sin \varepsilon_{2}=-\left(I_{z}+J_{z}\right) \bar{r} \omega-N_{\beta} \bar{\beta}
\end{aligned}
$$

If $m+m_{y}, m_{y} \alpha, Y_{\beta}$ and $N_{\beta}$ are given by the pure sway test, we can get $I_{z}+J_{z}, Y_{r}, N_{r}$ and- $\left(m+m_{x}\right)$ $\times U+Y_{r}$. Ship motions given by (i), (ii) and (iii) are shown in Fig. 2 .

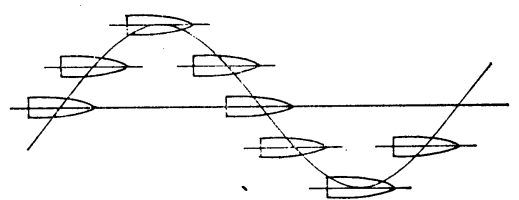

Pure Swaying Motion

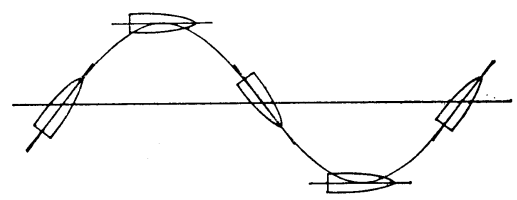

Pure Yawing Motion

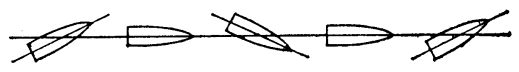

Combined Motion

Fig. 2 Definition of Motions

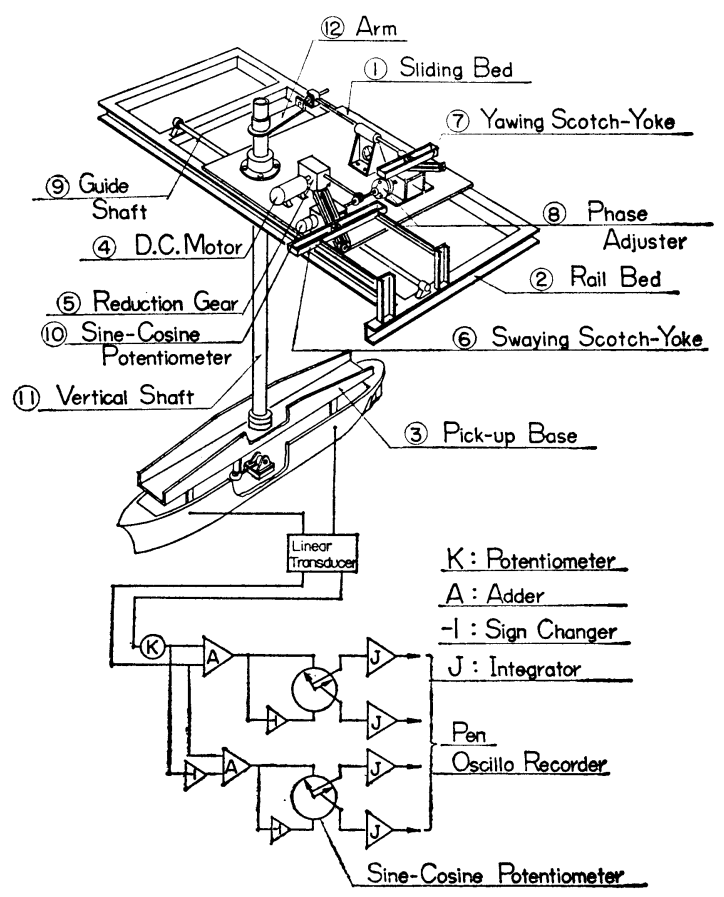

Fig. 3 Forced Yawing Apparatus and Phase Analyser

\section{Yawing oscillator}

The set-up of the yawing oscillator is as shown in Fig.3. In Fig. 3, a ship model attached to (3) is forced to yaw by the periodical rotation of the vertical shaft (11). The model is forced to sway simultaneously as the bed (1) slides transversely. The whole set-up is mounted on the towing carriage, and the yaw and sway motions are given by scotch yokes (7) and (6). Maximum swaying amplitude is $350 \mathrm{~mm}$ and the max yaw amplitude is $40^{\circ}$.

To give a "pure swaying motion", (6) only is adjusted to a specified stroke while (7) is adjusted to be zero. If the stroke of (6) is denoted $\eta_{s}$, the $\bar{\beta}$ in the equation (2) is expressed as follows :

$$
\bar{\beta}=\frac{\eta_{s} \omega}{U}
$$

To give a"pure yawing motion", it is necessary to adjust the phase between scotch yokes (7) and (6). Therefore a phase adjuster (8) was provided for this purpose. In the case of pure yawing motion, strokes $\eta_{s}$ and $\eta_{y}$ of the scotch yokes (6) and (7) should satisfy the following relation :

$$
\frac{\eta_{s} \omega}{U}=\frac{\eta_{y}}{a}
$$

Where $a$ is the arm length of (12) in Fig. 3. To give a combined motion, only (7) is adjusted to be specified stroke while the eccentricity of (6) is kept zero. 


\section{Measuring system}

To eliminate the high frequency noise which may be contained in the measured force or moment, a harmonic analysis technique similar to the one used by Van Leeuwen and Tanaka ${ }^{6}$ ) is employed.

Side forces $Y_{1}$ and $Y_{2}$ acting to the model at the points forward and after the C. G. of the model by distance $l$ are picked up by linear transformers.

Then Side force $\quad Y_{0}=Y_{1}+Y_{2}$

Moment $\quad N_{0}=l\left(Y_{1}-Y_{2}\right)$

are produced through an analog computor.

These $Y_{0}$ and $N_{0}$ are fed as the input to sine-cosine-potentiometers synchronized with the ship motion.

Then we get $Y_{0} \cos \omega t$

$Y_{0} \sin \omega t$

$N_{0} \cos \omega t$

$N_{0} \sin \omega t$

as outputs.

Integrating these values by an analog computor, we get for instance;

$$
\int \bar{Y}_{0} \cos \left(\omega t-\varepsilon_{1}\right) \cos \omega t d t=\frac{\bar{Y}_{0} \cos \varepsilon_{1}}{2} t+\frac{\bar{Y}_{0}}{4 \omega} \sin \left(2 \omega t-\varepsilon_{1}\right)
$$

(10) is a straight line of tangent $\frac{\bar{Y}_{0} \cos \varepsilon_{1}}{2}$ superposed by a sinusoidal curve of frequency $2 \omega$. Since the term we like to obtain is $\bar{Y}_{0} \cos \varepsilon_{1}$ as shown in equation (4), twice of the tangent of the mean curve of (10) gives the answer. In a similar way, we get

$$
\bar{Y}_{0} \sin \varepsilon_{1}, \bar{N}_{0} \cos \varepsilon_{2}, \text { and } \bar{N}_{0} \sin \varepsilon_{2} \text {. }
$$

Therefore, we can get the stability derivatives by equation (4), (7) and (9).

\section{Results obtained}

A $2 \mathrm{~m}$ model of series $60, C_{b}=0.60$ was used. Dimensions of the model are shown in Table 1 . The radius of gyration $K$ of the model is adjusted to 0.20 of ship length to get more accurate value of ship's added moment of inertia.

\begin{tabular}{|c|c|}
\hline Length Between Perpendiculars $L(\mathrm{~m})$ & 2.00 \\
\hline Breadth, $B(\mathrm{~m})$ & 0.267 \\
\hline Draft, $d(\mathrm{~m})$ & 0.107 \\
\hline Displacement, $V\left(\mathrm{~m}^{3}\right)$ & 0.03417 \\
\hline Block coefficient, $C_{b}$ & 0.600 \\
\hline Prismatic coefficient, $C_{p}$ & 0.614 \\
\hline Waterplane coefficient, $C_{w}$ & 0.706 \\
\hline Longitudinal center of buoyancy, $\%$ of $L$ from & $1.5 \mathrm{aft}$ \\
\hline Radius of gyration in air, $K L$ & $0.20 \mathrm{~L}$ \\
\hline Ratio of rudder area to projected area of the ship, $\%$ & 1.60 \\
\hline Aspect ratio & 1.90 \\
\hline Maximum thickness, $\%$ of chord length & 16.7 \\
\hline Position of Maximum thickess, $\%$ of chord length from leading edge & 35.0 \\
\hline Blade mumber & 4 \\
\hline Blade area ratio & 0.55 \\
\hline Dia $(\mathrm{cm})$ & 7.46 \\
\hline Pitch $(\mathrm{cm})$ & 8.02 \\
\hline Mean width ratio & 0.261 \\
\hline Blade thickness fraction & 0.045 \\
\hline Rake (deg) & 6.00 \\
\hline
\end{tabular}

Table 1 Characteristics of the Model

Stability derivatives are obtained of this model by the rotating arm technique at Stevens Institute of Technology7). 

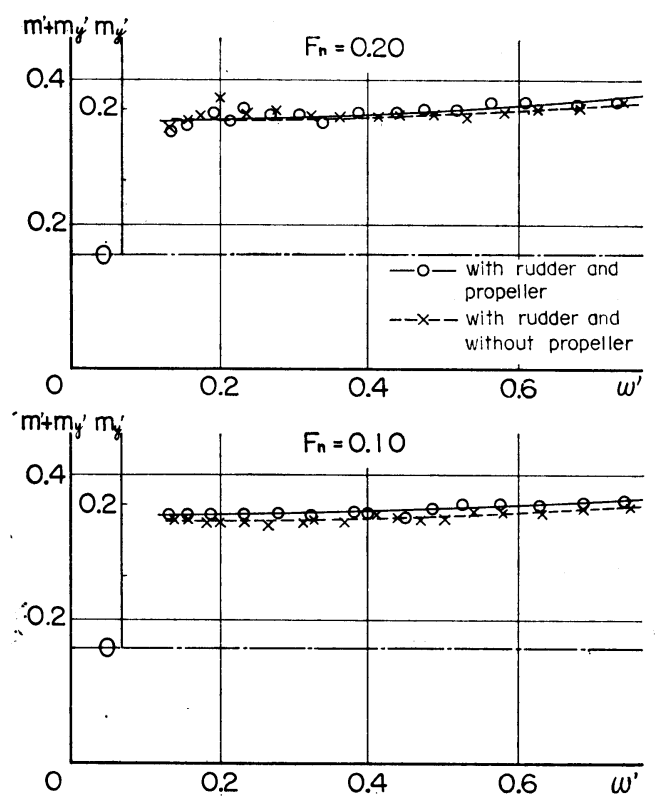

Fig. $4 m_{y}{ }^{\prime}-\omega^{\prime}$ curve
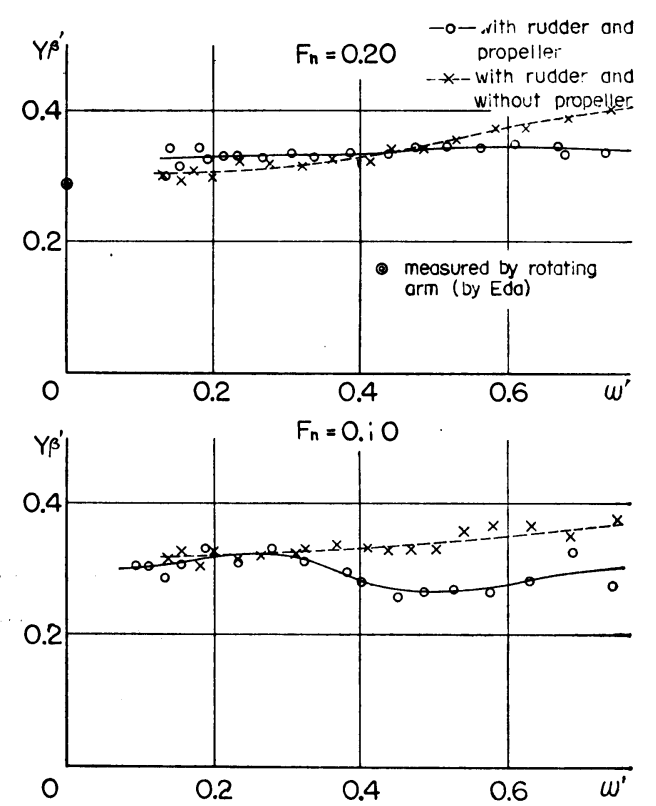

Fig. $5 Y_{\beta}^{\prime}-\omega^{\prime}$ curve
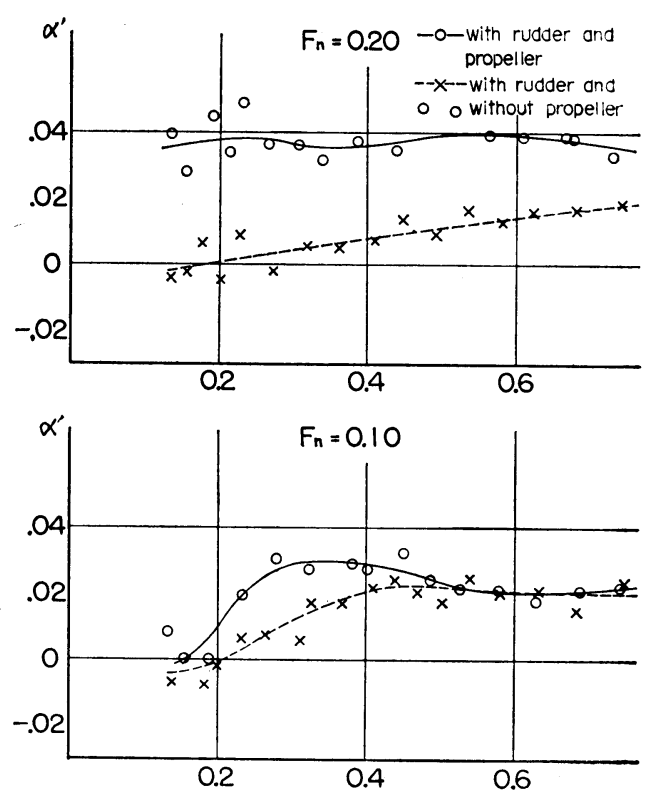

Fig. $7 \quad \alpha^{\prime}-\omega^{\prime}$ curve

Fig. $6 N_{\beta^{\prime}}-\omega^{\prime}$ curve 


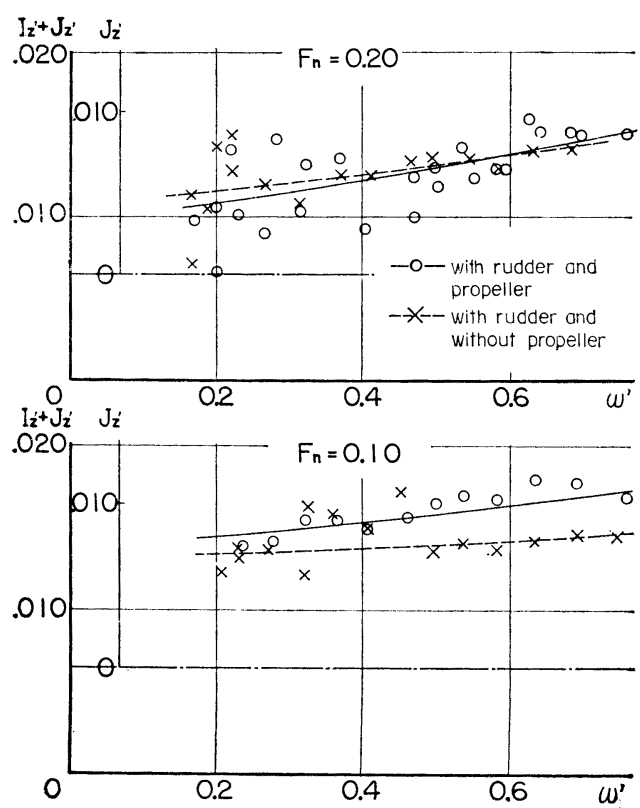

Fig. $8 J_{z}^{\prime}-\omega^{\prime \prime}$ curve
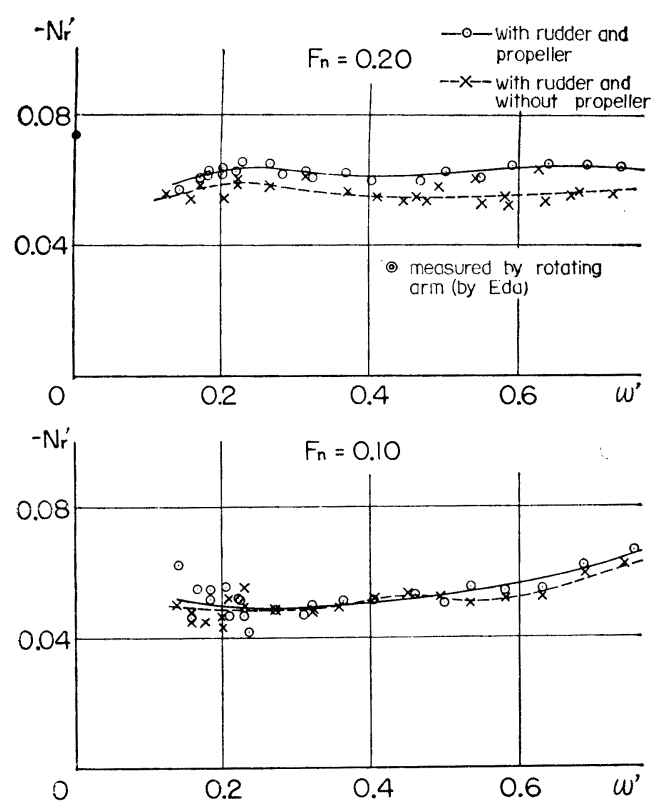

Fig. $10 N_{r}^{\prime}-\omega^{\prime}$ curve
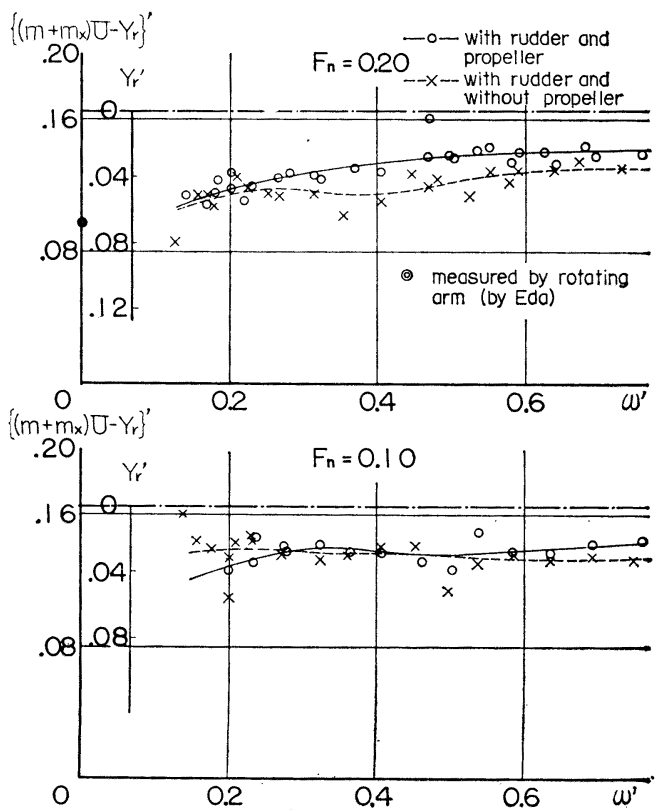

Fig. $9 \quad Y_{r}^{\prime}-\omega^{\prime}$ curve

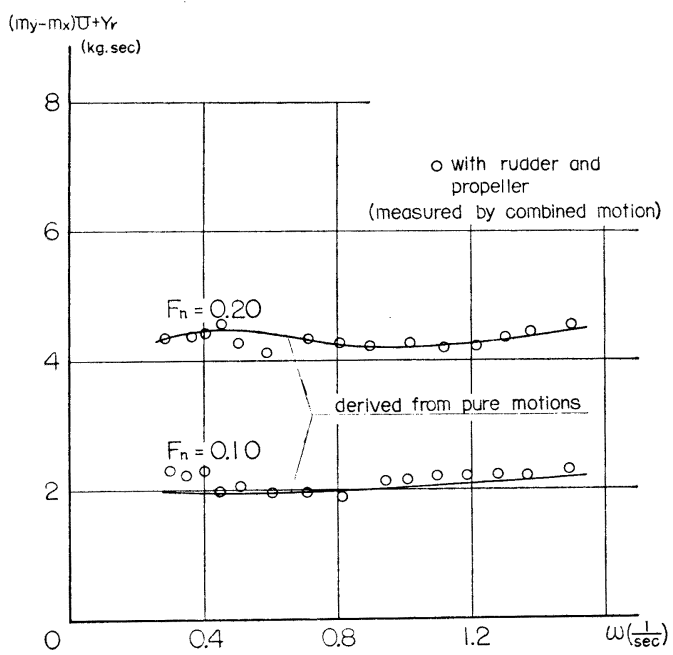

Fig. $11\left(m_{y}-m_{x}\right) U+Y_{r} \sim \omega$ curve 


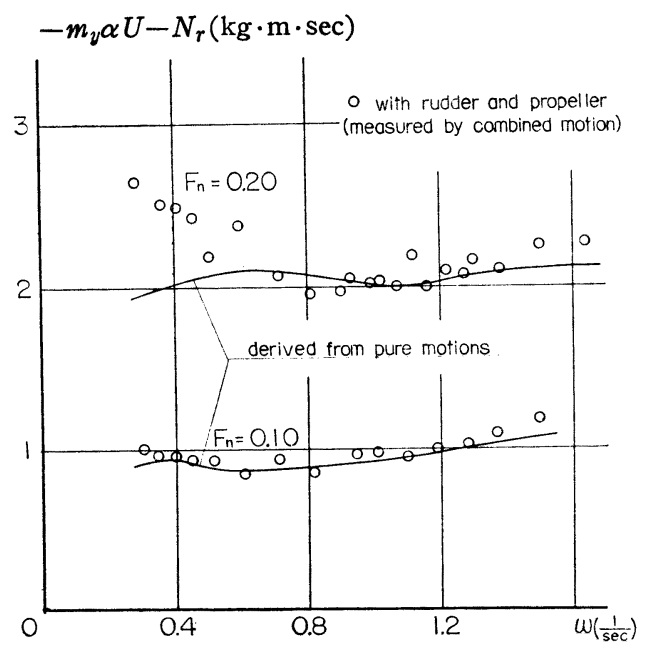

Fig. $12-m_{y} \alpha U-N_{r} \sim \omega$ curve

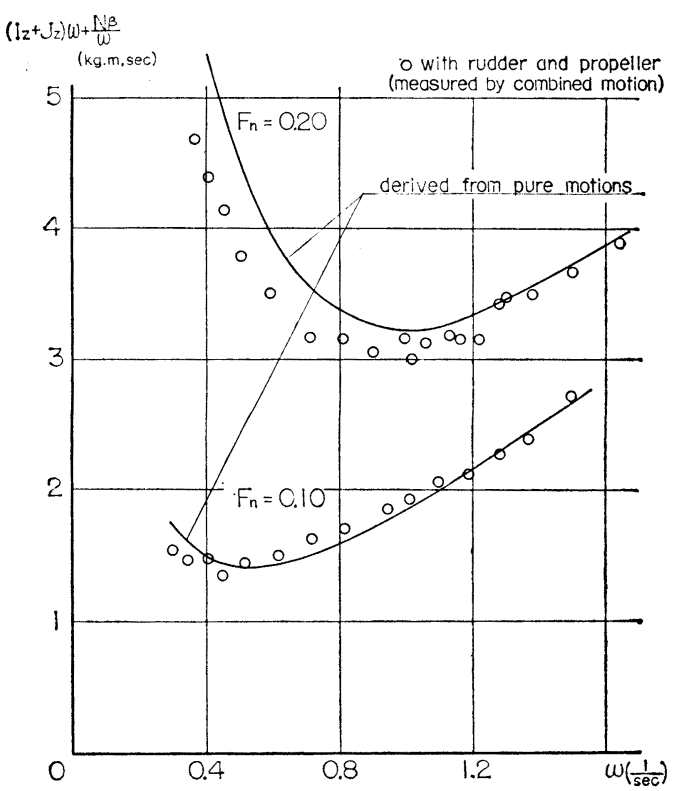

Fig. $13\left(I_{z}+J_{z}\right) \omega+N_{\beta} / \omega \sim \omega$ curve

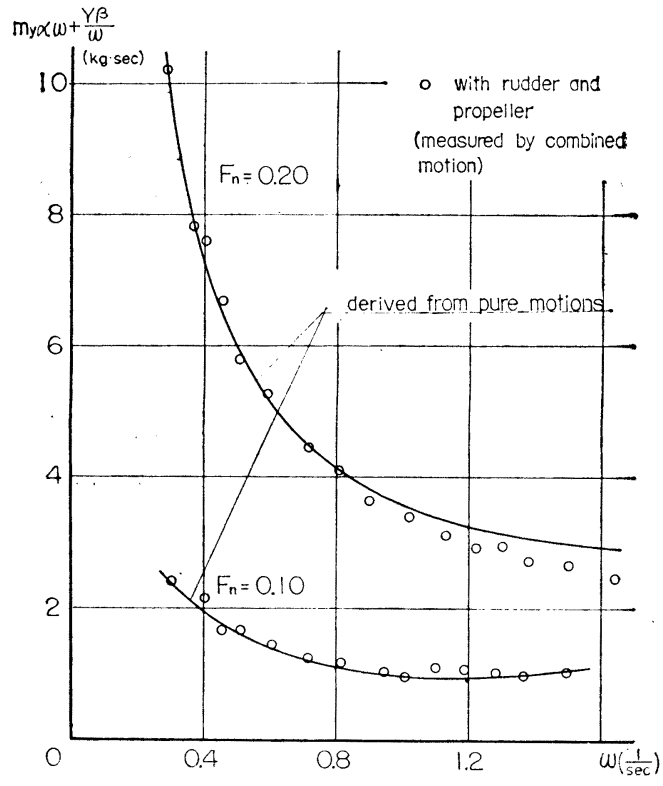

Fig. $14 m_{y} \alpha \omega+Y_{\beta} / \omega \sim \omega$ curve

For the condition of the model, two conditions-one is with rudder and without propeller and the other is with rudder and propeller-were chosen.

For the revolution of the propeller, a self propelling condition was chosen.

For the ship speed, $F_{n}=0.10$ and 0.20 were tested for each condition.

As to the magnitudes of $\beta$ and $r$, the following ranges were chosen to keep the motion in a liner range.

$$
\begin{aligned}
& \bar{\beta}^{\prime}<10^{\circ} \\
& \overline{\boldsymbol{r}}^{\prime}=\overline{\boldsymbol{r}} \frac{L}{U}<0.30
\end{aligned}
$$

Stability derivatives obtained are as shown in Figs. $4 \sim 14$, where symbols mean :

$$
\omega^{\prime}=\omega \sqrt{\frac{L}{g}} \quad \alpha^{\prime}=\frac{\alpha}{L}
$$




$$
\left.\begin{array}{ll}
m^{\prime}+m_{y}{ }^{\prime}=\frac{m+m_{y}}{\frac{1}{2} \rho L^{2} d} & I_{z}{ }^{\prime}+J_{z}{ }^{\prime}=\frac{I_{z}+J_{z}}{\frac{1}{2} \rho L^{4} d} \\
Y_{\beta^{\prime}}=\frac{Y_{\beta}}{\frac{1}{2} \rho L d U^{2}} & Y_{r}{ }^{\prime}=\frac{Y_{r}}{\frac{1}{2} \rho L^{2} d U} \\
N_{\beta^{\prime}}=\frac{N_{\beta}}{\frac{1}{2} \rho L^{2} d U^{2}} & N_{r}{ }^{\prime}=\frac{N_{r}}{\frac{1}{2} \rho L^{3} d U}
\end{array}\right\}
$$

As it is expected that the value of a stability derivative at zero frequency should coincide with the value of the same derivative obtained from the rotating arm technique, curves of the derivatives vs. frequency were extrapolated to zero frequency, and the values at zero frequency thus obtained were compared with ones which were obtained by rotating arm tests at Stevens Institute of Technology?) as shown in Table 2 .

Table 2 Comparison of the Stability Derivatives

\begin{tabular}{lccccccc}
\hline & $m_{y}^{\prime}$ & $Y_{\beta^{\prime}}$ & $N_{\beta^{\prime}}$ & $\alpha^{\prime}$ & $J_{z^{\prime}}$ & $Y_{r^{\prime}}$ & $N_{r^{\prime}}$ \\
Eda & - & 0.290 & 0.100 & - & - & 0.067 & -0.074 \\
Present data & 0.195 & 0.330 & 0.115 & 0.04 & 0.0040 & 0.058 & -0.060 \\
Leeuwen & 0.201 & 0.388 & 0.100 & 0.04 & 0.0088 & 0.067 & -0.060 \\
\hline
\end{tabular}

From Table 2, it can be said that values of derivatives obtained by the rotating arm technique and the present method agree fairly well. A reasonable agreement between the derivatives for $C_{b}=0.70$ model obtained by Van Leeuwen and for $C_{b}=0.60$ model is also recognized. In Figs $4 \sim 14$, it can be seen that the data points obtained from pure yawing motions show more scatter than the ones obtained from pure swaying motions. This scatter of data obtained from pure yawing motion mainly comes from the fact that a perfect pure yawing motion is very difficult to realize; i. e. it is very difficult to satisfy the relation $\frac{\eta_{s} \omega}{U}=\frac{\eta_{y}}{a}$ perfectly. A small changes in $U$ or $\omega$ will cause the deviation from $\beta=0$, and consequently, the effect of $\beta$ may be contained in the data as the error.

In examining the possible error caused by existing $\beta$, it will be easily seen that the effect of $\beta$ is most apparent for the measurement of $I_{z}+J_{z}$ in low frequecy range. Therefore, it will be wiser to detect the actual value of $\beta$ and correct the measured values using $m_{y}, Y_{\beta}$ and $N_{\beta}$ which are obtained by pure swaying motion.

As stated in Chapter 2, all the derivatives will be obtained from a set of two out of three kinds of motions, i.e., pure swaying motion, pure yawing motion, and combined motion. To check the reliability of this technique, values of $\left(m_{y}-m_{x}\right) U+Y_{r},-m_{y} \alpha U-N_{r}, \quad\left(I_{z}+J_{z}\right) \omega+\frac{N_{\beta}}{\omega}$ and $m_{y} \alpha \omega+\frac{Y_{\boldsymbol{\beta}}}{\omega}$ are calculated from the derivatives obtained from a set of pure swaying motion and pure yawing motion, and are compared with those which are obtained by combined motion as shown in Figs. $11 \sim 14$. Reasonable agreement will be recognized in those figures. This shows that we can pick up any combination of two out of three kinds of motions.

In view of difficulties in adjusting for the pure yawing motion, it will be wiser to choose a combination of pure swaying motion and combined motion except for very low frequencies where hydrodynamic moment caused by swaying is very large compared with inertia moment.

\section{Conclusions}

Summarizing the discussion, it can be stated :

(1) It was found that the forced yawing technique combined with harmonic analysis can produce reliable data of stability derivatives even in low frequency range.

(2) Stability derivatives obtained by the forced yawing technique as extrapolated to zero frequency 
agree very well with the ones obtained by the rotating arm technique.

(3) The added mass, added moment of inertia and the stability derivatives are obtained from a combination of two out of three kinds of motions; i. e., pure swaying motion, pure yawing motion, and combined moton. And it was found that a combination of pure swaying motion and combined motion is easy to conduct and also produce more accurate data, except at very low frequency.

\section{Acknowledgement}

The authors like to acknowledge the usefull helps given by Prof. Inui and his staff in conducting experiment, and usefull advice given by Dr. Tanaka of Ship Research Institute.

\section{Refierences}

1) Goodman A : "Experimental Technique and Methods of Analysis Used in Submerged Body Research." Third Symposium on Naval Hydrodynamics, Scheveningen, Holland, 1960

2) Motora S. \& R. B. Couch : “Maneuverability of Full Bodied Ships in Restricted Waters." Presented before the Fall Meeting of the Great Lakes and Great River Section of the SNAME, Oct., 1961

3) Paulling R: “The Dynamic Problem of Two Ships Operating on Parallel Courses in Close Proximity." University of California, July 1962

4) Van Leeuwen G. : "The Lateral Damping and Added Mass of an Oscillating Ship Model” Technological University-Delft Pub. No. 23, June 1964

5) Motora S. : "Forced Yawing Technique to obtain the Stability Derivatives as Frequency Dependents" Appendix 3 of the Comm. Report. 10 th ITTC Sept. 1963

6) Tanaka H. \& Kitagawa H. : "On the Study of Characteristics of Ship Motions by a Forced Oscillation Method.” Journal of Zosen Kiokai, Vol.111, June 1962

7) Eda H. \& C.L. Crane : "Steering Characteristics of the Series $60\left(C_{b}=60\right)$ " Davidson Laboratory Report 923, Oct. 1962 